\title{
Fate of remnant sinuses of Valsalva in patients with bicuspid and trileaflet valves undergoing aortic valve, ascending aorta, and aortic arch replacement
}

\author{
Rita Karianna Milewski, MD, PhD, Andreas Habertheuer, MD, PhD, Joseph E. Bavaria, MD, \\ Mary Siki, BS, Wilson Y. Szeto, MD, Eric Krause, MD, Varun Korutla, \\ Nimesh D. Desai, MD, PhD, and Prashanth Vallabhajosyula, MD, MS
}

\begin{abstract}
Objective: In patients presenting with aortic valvulopathy with concomitant ascending aortic aneurysm, surgical management of the sinus of Valsalva segment remains undefined, especially for moderately dilated aortic roots. In patients with this pathology undergoing aortic valve replacement with supracoronary ascending aorta replacement, we assessed the fate of the remnant preserved sinus of Valsalva segment stratified by aortic valve morphology and pathology.
\end{abstract}

Methods: From 2002 to 2015, 428 patients underwent elective aortic valve replacement with supracoronary ascending aorta replacement. Patients were stratified on the basis of valvular morphology (bicuspid aortic valve $[n=254]$ and tricuspid aortic valve $[\mathrm{n}=174]$ ), valvular pathology (bicuspid aortic valve with aortic stenosis $[\mathrm{n}=178]$, bicuspid aortic valve with aortic insufficiency [ $\mathrm{n}=76]$, tricuspid aortic valve with aortic stenosis $[\mathrm{n}=61]$, tricuspid aortic valve with aortic insufficiency $[\mathrm{n}=113])$, and preoperative sinus of Valsalva dimensions $(<40,40-45,>45 \mathrm{~mm})$.

Results: Kaplan-Meier analysis revealed no significant difference in freedom from reoperation in tricuspid aortic valve versus bicuspid aortic valve $(P=.576)$. Multivariable Cox regression model performed with sinus of Valsalva dimensions at baseline and follow-up as time-varying covariates did not adversely affect survival. A repeated-measure, mixed-effects model constructed to assess longitudinal sinus of Valsalva trends revealed that the retained sinus of Valsalva dimensions remain stable over long-term follow-up (discharge to $\geq 10$ years), irrespective of valvular morphology/pathology (bicuspid aortic valve with aortic insufficiency, tricuspid aortic valve with aortic insufficiency, tricuspid aortic valve with aortic stenosis) and preoperative sinus of Valsalva groups $(<40,40-45,>45 \mathrm{~mm})$.

Conclusions: In patients with nonaneurysmal sinuses of Valsalva undergoing aortic valve replacement with supracoronary ascending aorta replacement, the sinus segment can be preserved irrespective of the type of valvular pathology (aortic stenosis vs aortic insufficiency) or valvular morphology (bicuspid aortic valve vs tricuspid aortic valve). Aortic valve replacement with supracoronary ascending aorta replacement may have a stabilizing effect on the sinus segment over longterm follow-up in patients with tricuspid aortic valves or bicuspid aortic valves. (J Thorac Cardiovasc Surg 2017;154:421-32)

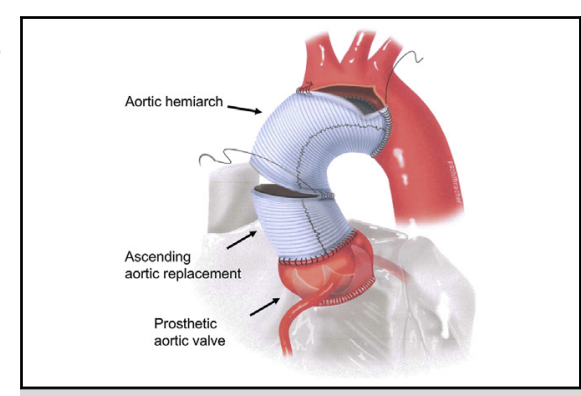

Aortic valve replacement and supracoronary ascending aortic replacement/hemiarch procedure.

Central Message

An aortic valve with supracoronary aortic replacement provides long-term stability to the remnant SOV segment in patients with bicuspid and tricuspid aortopathies.

\section{Perspective}

In patients undergoing aortic valve and supracoronary aortic replacement, a direct comparative analysis of the retained sinus segment in patients with BAV and TAV is lacking. We assess the long-term stability of the preserved sinus stratified by aortic valve morphology (bicuspid vs tricuspid) and pathology (AS or AI) and sinus dimensions to create evidencebased guidelines for the management of the sinus segment in patients with BAV or TAV aortopathies.

See Editorial Commentary page 433.

See Editorial page 419.

\footnotetext{
From the Department of Surgery, Hospital of the University of Pennsylvania, Philadelphia, $\mathrm{Pa}$.

Read at the 42nd Annual Meeting of The Western Thoracic Surgical Association, June 22-25, 2016, Waikoloa, Hawaii.

Received for publication June 27, 2016; revisions received Feb 26, 2017; accepted for publication March 26, 2017; available ahead of print June 7, 2017.

Address for reprints: Prashanth Vallabhajosyula, MD, MS, Hospital of the University of Pennsylvania, 3400 Spruce St, Philadelphia, PA 19104 (E-mail: Prashanth. vallabhajosyula@uphs.upenn.edu).

$0022-5223 / \$ 36.00$

Copyright (c) 2017 by The American Association for Thoracic Surgery

http://dx.doi.org/10.1016/j.jtcvs.2017.03.150
}

Bicuspid aortic valve (BAV) is the most common congenital valvular pathology with an incidence of $1 \%$ to $2 \%$ and a

Scanning this QR code will take you to a video for this article.

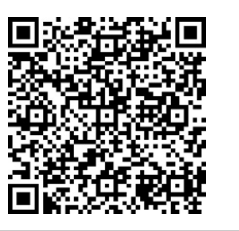




$$
\begin{array}{ll}
\text { Abbreviations and Acronyms } \\
\text { AI } & =\text { aortic insufficiency } \\
\text { AS } & =\text { aortic stenosis } \\
\text { AVRSCAAR } & =\text { aortic valve and supracoronary } \\
& \text { ascending aortic replacement } \\
\text { BAV } & =\text { bicuspid aortic valve } \\
\text { CI } & =\text { confidence interval } \\
\text { HR } & =\text { hazard ratio } \\
\text { NYHA } & =\text { New York Heart Association } \\
\text { SOV } & =\text { sinus of Valsalva } \\
\text { STJ } & =\text { sinotubular junction } \\
\text { TAV } & =\text { tricuspid aortic valve }
\end{array}
$$

known association with aortopathies. ${ }^{1-4}$ The incidence of ascending aortic aneurysms in patients with BAV is $30 \%$ to $50 \% .^{2-4}$ Tricuspid aortic valve (TAV) idiopathic aortopathies are primarily associated with older age, smoking, hypertension, and higher atherosclerotic burden.

It is well recognized that the presence of severe aortic root dilation with aortic valve disease requires root replacement in patients with TAV and BAV. What is less clear are the long-term characteristics and risk of aortic events for the retained sinus of Valsalva (SOV) in both BAV and TAV patients. An aortic valve replacement and supracoronary ascending aorta replacement (AVRSCAAR) procedure has been used for patients with a mild to moderately dilated sinus segment. This procedure has been used for patients with both TAV and BAV aortopathies and has the advantage of retaining the SOV and preserving the intact coronary ostia. On the basis of studies of the ascending aorta, it has been postulated that patients with BAV have a higher rate of complications ${ }^{5}$ of the remaining aorta and dilation of the proximal aorta and retained sinus segment. ${ }^{6}$ It has been shown that a nondilated BAV ascending aorta has degraded elastic lamella fibers compared with nondilated TAV ascending aorta. $^{7}$ However, a study by Heng and colleagues ${ }^{8}$ has suggested a more severe histologic abnormality in dilated TAV versus BAV aortas. Further extending these concepts, it has been suggested that the cause of aortic dilation is different in patients with aortic stenosis (AS) and aortic insufficiency (AI) and is based on TAV and BAV morphology, histology, and hemodynamic flow patterns. ${ }^{2,7,9}$ These findings prompted our investigation of the potential differential stability of the SOV between those with TAV and BAV with AS or AI.

To date, a direct comparative longitudinal study of the stability of the retained mildly to moderately dilated sinus segment in patients with BAV and TAV stratified by AS or AI valve pathology is lacking. We investigated the midterm outcomes of the preserved SOV segment stratified by aortic valve type and pathology.

\section{MATERIALS AND METHODS Patients}

This study was approved by the Institutional Review Board of the University of Pennsylvania. A prospectively maintained institutional database was retrospectively reviewed to stratify all patients by BAV or TAV valvular pathology with concomitant ascending aortic aneurysm who underwent AVRSCAAR. From 2002 to 2015, 428 patients underwent an elective AVRSCAAR for valvular and aneurysmal pathology. To maintain homogeneity within each group, patients with known connective tissue disorders, acute type A dissection, endocarditis, or reoperative procedures, and emergency cases were excluded. All aortoplasty procedures were excluded. An ascending hemiarch/total arch replacement procedure was performed in $100 \%$ of the cases included in this study.

To account for the heterogeneous disease processes of patients with BAV and TAV with aortic valvulopathy and ascending aneurysm, we stratified the patient population by 4 variables, including valve morphology (BAV vs TAV), valve pathology (AS vs AI), baseline SOV dimensions (mild [ $<40 \mathrm{~mm}$ ], moderate [40-45 mm], severe [ $>45 \mathrm{~mm}]$ ), and time relation to the procedure (preoperative, discharge, and longitudinal follow-up to $>10$ years). To eliminate the variable of a third cohort, all patients with AS and AI pathology were grouped into the AS subcategory. Our institutional algorithm for AVRSCAAR based on the dimension of the aortic root, the valvular pathology, and the degree of effacement (STJ/annular ratio) is delineated in Figure 1. The final decision on the procedure performed was based on intraoperative findings and the operating surgeon's decision in cases of $\mathrm{STJ} /$ annular ratio greater than 1.5 or thinning of the sinus segment. The distribution of the 428 patients meeting inclusion criteria by subgroups was as follows: BAV group $=254$ patients $(\mathrm{BAV}$ AS $=178, \mathrm{BAV} \mathrm{AI}=76)$; TAV group $=174$ patients $($ TAV AS $=61$, TAV AI $=113)$.

\section{Surgical Procedure}

In all cases, aortic valve replacement, ascending aorta replacement, and aortic arch reconstruction under hypothermic circulatory arrest were performed (Central Image).

Ascending aorta replacement. The ascending aorta was resected 1 to $1.5 \mathrm{~cm}$ above the sinotubular junction (STJ), and an appropriately sized Dacron graft (Vascutek, Glasgow, United Kingdom) was anastomosed to the aorta just above the STJ with a running Prolene suture (Video 1).

Aortic arch replacement. Deep hypothermic circulatory arrest was initiated using retrograde cerebral perfusion via the superior vena cava or antegrade cerebral perfusion via the axillary artery. An aggressive transverse hemiarch was tailored by resecting the majority of the lesser curvature of the aortic arch, and a straight Dacron graft was beveled and anastomosed to the remnant aortic arch with a running Prolene stitch as an open distal anastomosis. The aortic graft was cannulated to reinitiate cardiopulmonary bypass.

Total arch replacement was performed using deep hypothermic circulatory arrest with antegrade cerebral perfusion via the axillary artery or direct ostial arch vessel perfusion. The proximal descending thoracic aorta was anastomosed first to a 3- or 4-branch graft, and each head vessel was individually reimplanted to a branch of the graft. Typically, on completion of the distal aortic anastomosis and the left subclavian artery anastomosis, cardiopulmonary bypass was reinitiated and the left common carotid and innominate arteries were anastomosed individually.

Aortic valve replacement. On aggressive debridement of the valve and the annulus, valve replacement was performed by placing interrupted annular stitches circumferentially (Central Image).

\section{Clinical and Echocardiographic Follow-up}

Patients were seen in the Aorta Clinic and in individual surgeon clinics. In these patients, imaging and clinical follow-up were readily available. For patients not being followed routinely at our institution, phone calls were made to patients, their primary care physicians, and cardiologists to bring 


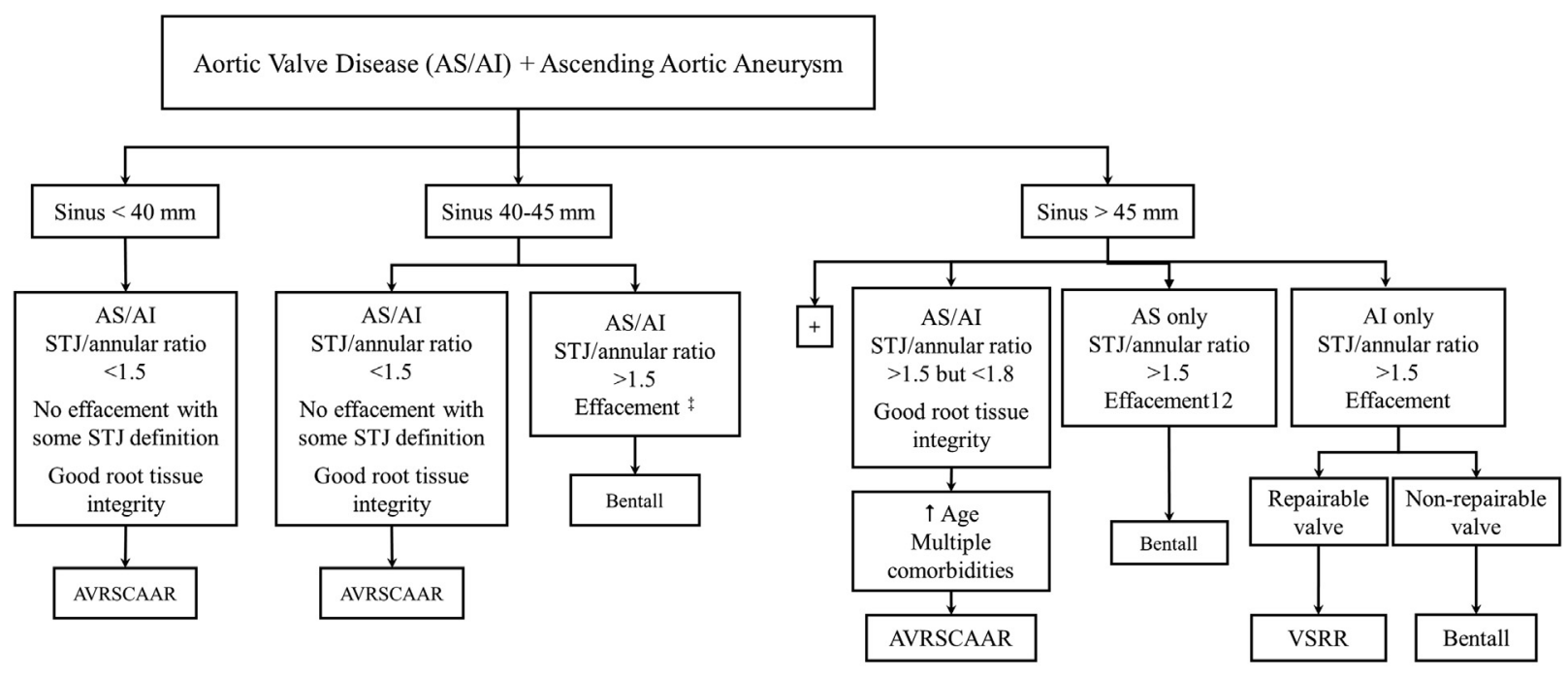

*no intermittent growth $>3-4 \mathrm{~mm}$ per year

$\ddagger$ If tissue thinning at time of surgery, then Bentall is indicated.

+ If AS/AI with STJ/annular ratio $<1.5$, then Bentall is indicated

unless there is increased age or multiple comorbidities

FIGURE 1. Institutional algorithm for ascending aortic aneurysm disease and AVRSCAAR procedure. AS, Aortic stenosis; $A I$, aortic insufficiency; $S T J$, sinotubular junction; AVRSCAAR, aortic valve and supracoronary ascending aortic replacement; VSRR, valve-sparing root reimplantation.

patients to the Aorta Clinic for follow-up evaluation. If patients were unable to return to clinic for personal reasons, with their permission, echocardiographic and clinical follow-up data were obtained from their primary care physicians and cardiologists. All patients with BAV and TAV were followed in the outpatient Aorta Clinic using transthoracic echocardiography exclusively.

\section{Statistical Analysis}

General statistics were analyzed using STATA/MP v14.2 (StataCorp LP, College Station, Tex) and SPSS v.23 (SPSS Inc, Armonk, NY).

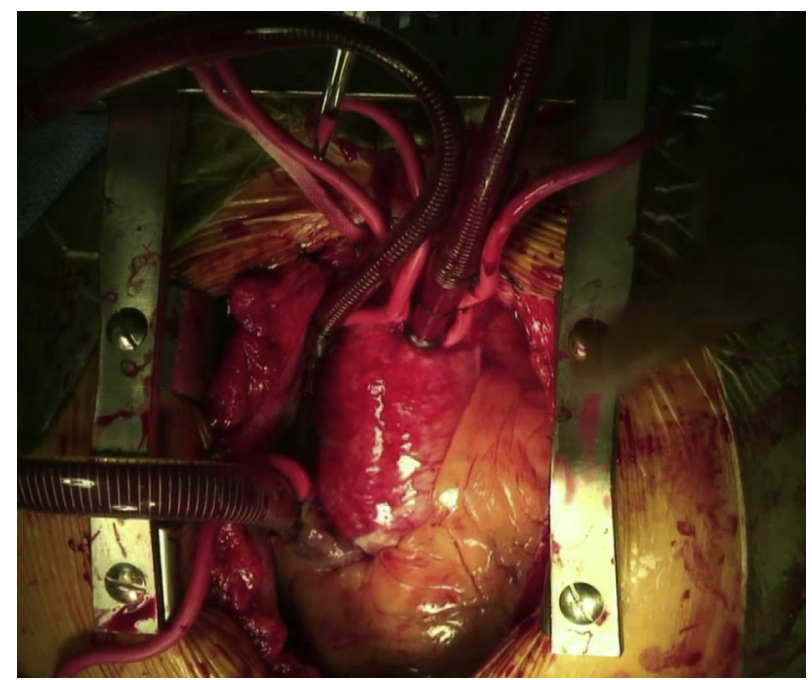

VIDEO 1. Intraoperative video demonstrating aortic valve repair with subvalvular aortic ring annuloplasty and supracoronary ascending aorta replacement with a Dacron graft using a hemiarch approach. Video available at: http://www.jtcvsonline.org/article/S0022-5223(17)30870-X/addons.
Predictive multivariate models and mixed-effect models for repeated measures were computed in STATA/MP v14.2, and R. Graphs were drawn in STATA/MP v14.2. First, data were checked for normality. Descriptive statistics were presented as means \pm standard deviation for continuous variables, median (interquartile range) for continuous variables in cases of non-normality, and percentage (frequency) for categoric variables. Continuous variables were compared using the unpaired $t$ test, paired dependent sample $t$ test in cases of repeated measures (preoperative vs early postoperative SOV diameters), 1-way analysis of variance, and Wilcoxon signed-rank test in cases of nonnormality. Fisher exact and chi-square statistics were used to compare categoric variables. All tests were 2 sided with the alpha level set at .05 for statistical significance.

A linear mixed-effects regression for repeated measures was fitted to validate predictors of SOV diameters over time and to analyze SOV trends over a longitudinal follow up. Preoperative valve morphology (TAV, BAV), valve pathology (AI, AS), preoperative SOV diameters (continuous variable), and postoperative measurement time points (continuous variable) were included as fixed effects, with the unique patient identification number as random effect. Measurement time points were included as repeatedmeasure time points. In addition, an interaction term of preoperative SOV diameters and postoperative measurement time points was introduced as a separate fixed effect covariate. A generalized linear model was used to describe predictors of the continuous response variable discharge/ 1-month SOV diameter.

Multivariable Cox regression was used to determine independent predictors of overall mortality. Factors included in the model were age, ascending aorta diameter (millimeters), time period of surgery (before or after 2009), gender, hypertension, New York Heart Association (NYHA) class III/IV, SOV greater than $45 \mathrm{~mm}$, and valve morphology (TAV, $\mathrm{BAV})$. To further determine the impact of longitudinal retained SOV trends on long-term outcome, follow-up SOV dimensions were added to the existing time-to-event model as a time-varying covariate. Kaplan-Meier method and log-rank statistics were used to determine and compare freedom from reoperation and reoperation-free survival (censoring for reoperation and death). Kaplan-Meier estimates and mean SOV diameters 
TABLE 1. Demographics and preoperative parameters

\begin{tabular}{|c|c|c|c|c|c|}
\hline \multirow[b]{2}{*}{ Variable } & \multicolumn{2}{|c|}{ TAV $(n=174)$} & \multicolumn{2}{|c|}{ BAV $(n=254)$} & \multirow[b]{2}{*}{$P$ value } \\
\hline & $\mathbf{A I}(n=113)$ & $\mathrm{AS}(\mathrm{n}=61)$ & $\mathbf{A I}(\mathbf{n}=76)$ & $\mathbf{A S}(\mathbf{n}=178)$ & \\
\hline \multicolumn{6}{|l|}{ Demographics } \\
\hline Age, y & $71.8 \pm 9.1$ & $72.1 \pm 8.3$ & $54.3 \pm 11.9$ & $61.6 \pm 10.8$ & $<.001$ \\
\hline Sex (male) & $60(53.1 \%)$ & $39(63.9 \%)$ & $65(85.5 \%)$ & $132(74.2 \%)$ & $<.001$ \\
\hline Hypertension & $95(84.1 \%)$ & $52(85.2 \%)$ & $50(65.8 \%)$ & $118(66.3 \%)$ & $<.001$ \\
\hline Smoking & $17(15.0 \%)$ & $14(23.0 \%)$ & $12(15.8 \%)$ & $23(12.3 \%)$ & .256 \\
\hline Dyslipidemia & $70(61.9 \%)$ & $47(77.0 \%)$ & $33(43.4 \%)$ & $106(59.6 \%)$ & .008 \\
\hline Diabetes mellitus & $17(15.0 \%)$ & $12(19.7 \%)$ & $3(3.9 \%)$ & $24(13.5 \%)$ & .065 \\
\hline \multicolumn{6}{|l|}{ No. of diseased vessels } \\
\hline 1 -vessel disease & $16(14.2 \%)$ & $11(18.0)$ & $4(5.3 \%)$ & $25(14.0 \%)$ & \\
\hline 2-vessel disease & $13(11.5)$ & $10(16.4 \%)$ & $3(3.9 \%)$ & $12(6.7 \%)$ & $<.001$ \\
\hline 3-vessel disease & $16(14.2)$ & $17(27.9)$ & $2(2.6)$ & $8(4.5 \%)$ & \\
\hline Aortic coarctation & $0(-)$ & $0(-)$ & $0(-)$ & $4(2.2 \%)$ & .096 \\
\hline LVEF < $<0 \%$ & $14(12.4 \%)$ & $8(13.1 \%)$ & $4(5.3 \%)$ & $10(5.6 \%)$ & .010 \\
\hline LVEF \% & $53.8 \pm 13.6$ & $55.1 \pm 16.2$ & $55.8 \pm 12.6$ & $60.2 \pm 12.2$ & .002 \\
\hline NYHA class III/IV & $33(29.2 \%)$ & $25(41.0 \%)$ & $9(11.8 \%)$ & $37(20.8 \%)$ & $<.001$ \\
\hline \multicolumn{6}{|l|}{ Echocardiographic data } \\
\hline Stenotic valve pathology & - & $61(35.1 \%)$ & - & $178(70.1 \%)$ & $<.001$ \\
\hline Insufficiency valve pathology & $113(64.9 \%)$ & - & $76(29.9 \%)$ & - & $<.001$ \\
\hline
\end{tabular}

over long-term follow-up were homogenously plotted with $95 \%$ confidence interval (CI) bands/bars.

\section{RESULTS}

Demographics, Preoperative Parameters, and Intraoperative and Postoperative Outcomes

Demographics and preoperative parameters are listed in Table 1. Mean age was $59.4 \pm 11.6$ years and $71.9 \pm 8.8$ years $(P<.001)$ for patients with BAV and TAV, respectively $(77.6 \%$ vs $56.9 \%$ male gender). There was a significantly higher subpopulation of AS in the $\mathrm{BAV}$ cohort $(\mathrm{BAV} \mathrm{AS}=70.1 \%$ vs $\mathrm{TAV} \mathrm{AS}=35.1 \%$, $P<.001)$. The TAV cohort of patients had a higher incidence of heart failure (NYHA III/IV $=33.3 \%$ vs $18.1 \%$, $P<.001)$ and an increased incidence of dyslipidemia $(67.2 \%$ vs $54.7 \%, P=.008)$. There was increased atherosclerotic burden noted in the ascending aorta at operation and increased incidence of diseased coronary vessels in patients with TAV $(P<.001)$ (Table 1). Preoperative root diameters and stratification of patients with BAV and TAV by SOV dimensions are shown in Table 2. Overall, the preoperative SOV dimensions for TAV and BAV cases were similar.
Intraoperative results. Intraoperative data are detailed in Table 3. AVRSCAAR was performed using a straight Dacron graft in $100 \%$ of cases. Mechanical valves were used in $2.3 \%$ of TAV cases $(97.7 \%$ biologic) and $5.1 \%$ of BAV cases ( $94.9 \%$ biologic). Cardiopulmonary bypass times $(219.8 \pm 64.0$ minutes vs $195.6 \pm 54.9$ minutes, $P<.001)$ and aortic crossclamp times $(159.2 \pm 51.4$ minutes vs $143.1 \pm 39.9$ minutes, $P<.001)$ were significantly longer in the TAV cohort, most likely because of the increased number of diseased coronary vessels in those with TAV.

Postoperative outcomes. Postoperative outcomes are listed in Table 4. In-hospital/30-day mortality was higher in patients with TAV $(5.2 \%$ [9/174] vs $1.6 \%$ in patients with BAV [4/254], $P=.033$ ). The incidence of transient ischemic attack/stroke was higher in the TAV group (3.4\% [6/174] vs $0.8 \%$ [2/254], $P=.04)$. In patients from both groups presenting with AI, significant left ventricular remodeling was noted on postoperative echocardiographic assessments compared with preoperative dimensions. Left ventricular end-diastolic dimension decrease of $7.4 \pm 1.0 \mathrm{~mm}(P<.001)$ was noted for those with TAV AI, and a mean decrease of

TABLE 2. Subgroup stratification by preoperative sinus of Valsalva dimensions and valve morphology

\begin{tabular}{llll}
\hline \multicolumn{1}{c}{ Variable } & \multicolumn{1}{c}{ TAV $(\mathbf{n}=\mathbf{1 7 4})$} & BAV $(\mathbf{n}=\mathbf{2 5 4})$ & $\boldsymbol{P}$ value \\
\hline Aortic root diameter $(\mathrm{mm})$ & $37.8 \pm 5.9(\mathrm{n}=155)$ & $37.4 \pm 4.6(\mathrm{n}=199)$ & .531 \\
Group A $(<40 \mathrm{~mm})$ & $34.1 \pm 3.7(\mathrm{n}=93)$ & $34.8 \pm 3.4(\mathrm{n}=129)$ & .128 \\
Group B $(40-45 \mathrm{~mm})$ & $41.7 \pm 1.9(\mathrm{n}=48)$ & $41.9 \pm 1.5(\mathrm{n}=66)$ & .519 \\
Group C $(>45 \mathrm{~mm})$ & $48.8 \pm 3.5(\mathrm{n}=14)$ & $46.8 \pm 1.5(\mathrm{n}=4)$ & .289 \\
\hline
\end{tabular}

Data are presented as mean $\pm \mathrm{SD}$. $T A V$, Tricuspid aortic valve; $B A V$, bicuspid aortic valve. 
TABLE 3. Intraoperative data

\begin{tabular}{lccr}
\hline \multicolumn{1}{c}{ Variable } & TAV $(\mathbf{n}=\mathbf{1 7 4})$ & BAV $(\mathbf{n}=\mathbf{2 5 4})$ & $\boldsymbol{P}$ value \\
\hline Cardiopulmonary bypass (min) & $219.8 \pm 64.0$ & $195.6 \pm 54.9$ & $<.001$ \\
Aortic crossclamp (min) & $159.2 \pm 51.4$ & $143.1 \pm 39.9$ & $<.001$ \\
Mechanical valve & $4(2.3 \%)$ & $13(5.1 \%)$ & .142 \\
Biological valve & $170(97.7 \%)$ & $241(94.9 \%)$ & .142 \\
Valve prosthesis size (mm) & $24.5 \pm 2.2$ & $25.9 \pm 2.1$ & $<.001$ \\
\hline
\end{tabular}

Data are presented as mean $\pm \mathrm{SD}$ and $\mathrm{n}(\%) . T A V$, Tricuspid aortic valve; $B A V$, bicuspid aortic valve.

$6.1 \pm 1.0 \mathrm{~mm}(P<.001)$ was noted in the BAV AI subgroup (data not shown).

\section{Longitudinal Follow-up}

In total, 428 patients met inclusion criteria with total follow-up of 1672 patient-years.

Sinus of Valsalva measurement. There was no significant difference in mean preoperative aortic root diameters between BAV and TAV cohorts for the AS or AI subpopulations: TAV AS $36.0 \pm 6.1 \mathrm{~mm}$, TAV AI $38.7 \pm 5.6 \mathrm{~mm}, \mathrm{BAV}$ AS $36.6 \pm 4.7 \mathrm{~mm}$, and BAV AI $39.2 \pm 3.9 \mathrm{~mm}(P=.531$ on analysis of variance) (Table 2). The number of patients remaining in the analysis for every follow-up time point is shown in Figure 2. Longitudinal follow-up data for SOV dimensions stratified into 12 subgroups based on valve morphology (BAV vs TAV), valve pathology (AS vs AI), and preoperative SOV dimension ( $<40 \mathrm{~mm}$ vs $40-45 \mathrm{~mm}$ vs $>45 \mathrm{~mm}$ ) are delineated in Figure 2.

A dependent-sample paired $t$ test for the stratified TAV AS, TAV AI, BAV AS, and BAV AI subpopulations comparing preoperative with early postoperative (discharge to 1 month) echocardiographic assessments of SOV diameters revealed an early, significant decrease in retained sinus dimensions $(38.2 \pm 5.3 \mathrm{~mm}$ vs $35.1 \pm 5.8 \mathrm{~mm}, P<.001)$. Results of a generalized linear model to determine the predictors of early postoperative reduction in SOV dimensions are shown in Table 5. Although patient age and aortic valvular morphology/pathology had no discernible effect on early SOV dimensions, larger preoperative SOV diameters were associated with greater early postoperative reduction in SOV dimensions $(P=.030)$.

Repeated-measure mixed effects model for longitudinal echocardiographic assessment of sinus of Valsalva. To

TABLE 4. Postoperative outcomes

\begin{tabular}{lccr}
\hline \multicolumn{1}{c}{ Variable } & $\begin{array}{c}\text { TAV } \\
(\mathbf{n}=\mathbf{1 7 4})\end{array}$ & $\begin{array}{c}\text { BAV } \\
(\mathbf{n}=\mathbf{2 5 4})\end{array}$ & $\begin{array}{c}\boldsymbol{P} \\
\text { value }\end{array}$ \\
\hline Stroke/transient ischemic attack & $6(3.4 \%)$ & $2(0.8 \%)$ & .046 \\
Renal failure & $6(3.4 \%)$ & $1(0.4 \%)$ & .014 \\
Renal failure requiring dialysis & $3(1.7 \%)$ & $1(0.4 \%)$ & .160 \\
Prolonged ventilation $(>24 \mathrm{~h})$ & $50(28.7 \%)$ & $27(10.6 \%)$ & $<.001$ \\
In-hospital/30-d mortality & $9(5.2 \%)$ & $4(1.6 \%)$ & .033 \\
\hline
\end{tabular}

Data are presented as mean \pm SD and $\mathrm{n}(\%) . T A V$, Tricuspid aortic valve; $B A V$, bicuspid aortic valve. achieve a statistically comprehensive interpretation incorporating long-term echocardiography data, we modeled a mixed-effects regression based on a repeated-measure approach in a longitudinal fashion. Valvular morphology and pathology at baseline, preoperative SOV diameter, postoperative time course, and interaction effect of preoperative SOV diameters and postoperative time course were used as covariates. Within-subject and within-stratified subgroup comparison failed to show main effects for follow-up time on the postoperative SOV pattern $(P=.935)$ (Table 6 and Figure 2), implying that the pattern in SOV trends was stable and sustained (discharge to $\geq 10$ years) irrespective of valvular morphology and pathology (BAV AI, BAV AS, TAV AI, and TAV AS) (Table 6 and Figure 2). This was statistically supported by the additional introduction of an interaction term (preoperative SOV diameter with postoperative time course), which also failed to show significant effects $(P=.783)$. Not surprisingly, preoperative SOV dimensions significantly affected the retained postoperative sinus dimensions $(P<.001)$. Taken together, the longitudinal data assessment implies that an initial and pronounced postoperative decrease in SOV dimensions occurs with AVRSCAAR independently of aortic valve morphology $(P=.155)$, aortic valve pathology $(P=.583)$, and age $(P=.573)$. This is then followed by a sustained and stable pattern of SOV dimension throughout the postoperative time course of 10 years or more in all the subgroups.

Multivariable Cox regression. The results of the Cox regression model to determine factors associated with mortality over long-term follow-up are shown in Table 7. Only increased age (hazard ratio [HR], 1.061; 95\% CI, 1.008$1.117 ; P=.022$ ) and advanced heart failure (NYHA classes III and IV; HR, 3.220; 95\% CI, 1.463-7.084; $P=.004$ ) were independently predictive of increased mortality. Of note, neither a dilated sinus segment at baseline $(>45 \mathrm{~mm})$ nor the type of valvular morphology (TAV vs BAV) had a discernible association with mortality (HR, 1.040; 95\% CI, 0.286-3.780; $P=.952$ and HR, 2.307; 95\% CI, 0.946-6.124; $P=.065$ ). There was an inverse effect for the year of surgical intervention, with a better survival for procedures performed during and after the year 2009 (HR, $0.275 ; 95 \%$ CI, $0.113-0.672 ; P=.005)$. A $10 \%$ increase (nonsignificant) in SOV dimensions as a time-varying covariate for the time-to-event model proved to have no predictive effect in terms of overall mortality. 


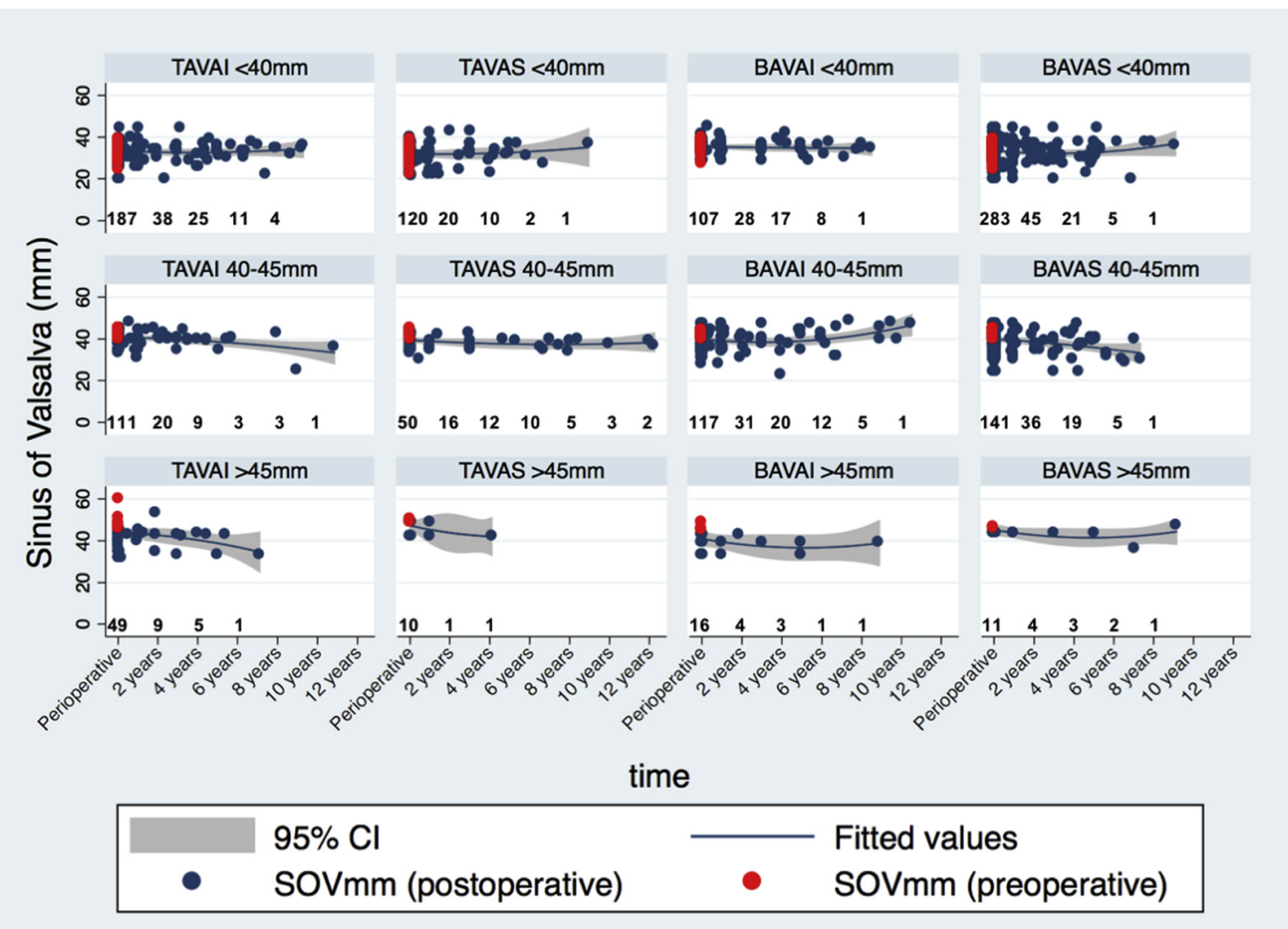

FIGURE 2. Long-term follow-up of SOV dimensions in patients with BAV and TAV disease with ascending aneurysm undergoing AVRSCAAR stratified by preoperative SOV dimensions. TAVAI, Tricuspid aortic valve with aortic insufficiency; TAVAS, tricuspid aortic valve with aortic stenosis; BAVAI, bicuspid aortic valve with aortic insufficiency; BAVAS, bicuspid aortic valve with aortic stenosis; $C I$, confidence interval; SOV, sinus of Valsalva.

\section{Aortic Reoperation and Long-Term Survival in Patients Undergoing Aortic Valve Replacement and Supracoronary Ascending Aorta Replacement}

Freedom from aortic reoperation in the BAV and TAV cohorts is delineated in Figure 3. Overall, both cohorts showed excellent long-term durability of the AVRSCAAR procedure, with 10-year freedom from aortic reoperation rates of $97 \%$ and $95 \%$ in the BAV and TAV subgroups, respectively. Reoperation-free survival, defined by freedom from aortic reoperation events and death, is shown for the BAV and TAV cohorts in Figure 4. This showed that the BAV group had improved reoperation-free survival compared with the TAV group $(P<.001$ by log rank). The type of valvular

TABLE 5. Generalized linear model showing predictors of postoperative reduction in sinus of Valsalva dimensions

\begin{tabular}{lccc}
\hline & Coefficient & SE & $P$ value \\
\hline Age & -0.005 & 0.009 & .573 \\
AI & -0.117 & 0.213 & .583 \\
TAV & -0.342 & 0.240 & .155 \\
Preoperative SOV diameter & -0.420 & 0.194 & .030 \\
\hline
\end{tabular}

$S E$, Standard error; $A I$, aortic insufficiency; $T A V$, tricuspid aortic valve; $S O V$, sinus of Valsalva. pathology within each group did not show a significant difference in reoperation-free survival (BAV AI vs BAV AS: $\log$ rank $P=.163$; TAV AI vs TAV AS: $\log$ rank $P=.285$ ) (Figure 4).

\section{DISCUSSION}

In patients with critical aortic valve disease and ascending aortic aneurysm, the management of the SOV segment for patients with a moderately dilated aortic root remains unresolved. Multiple studies have advocated AVRSCAAR as a treatment, thereby preserving the intact moderately dilated sinus segment and coronary ostia, ${ }^{10,11}$ whereas others suggest removal of the sinus segment and a Bentall root replacement procedure. ${ }^{12,13}$ However, most investigations have limited their analysis to the retention or replacement of the sinus segment of known congenital BAV aortopathies. There is limited direct comparative analysis regarding the stability of the remnant SOV segment between BAV and TAV cases. In this study, we assessed the fate of the remnant, preserved SOV segment in patients undergoing AVRSCAAR (Central Image).

Clinical reports on comparative histologic analysis of the aortic specimen have shown that the bicuspid aorta is 
TABLE 6. Repeated-measures, mixed-effect longitudinal model

\begin{tabular}{lccc}
\hline & Coefficient & SE & $P$ value \\
\hline Valve geometry and pathology & & & \\
$\quad$ BAV AI & Reference & & \\
BAV AS & -1.061 & 0.804 & .187 \\
$\quad$ TAV AI & -0.692 & 0.808 & .391 \\
$\quad$ TAV AS & -0.98 & 0.925 & .290 \\
$\begin{array}{l}\text { Preoperative SOV diameter } \\
\quad \text { (continuous) }\end{array}$ & 0.654 & 0.567 & $<.001$ \\
$\begin{array}{l}\text { Postoperative time course } \\
\quad \text { (continuous) }\end{array}$ & 0.03 & 0.367 & .935 \\
$\begin{array}{l}\text { Interaction term } \\
\text { (preoperative SOV diameter with time) }\end{array}$ & 0.003 & 0.009 & .783 \\
\hline
\end{tabular}

$S E$, Standard error; $B A V$, bicuspid aortic valve; $A I$, aortic insufficiency; $A S$, aortic stenosis; $T A V$, tricuspid aortic valve; $S O V$, sinus of Valsalva.

abnormal even if it is not aneurysmal. It is known that BAV is a multifaceted heterogeneous disease with an underlying genetic and developmental foundation. ${ }^{3,14-17}$ We now believe that both BAV and TAV aortopathies are heterogeneous disease processes with a common clinical presentation of a bicuspid or trileaflet aortic valve. Therefore, to understand the impact of a common therapeutic surgical procedure such as AVRSCAAR on the clinically important aspects of sinus segment size and geometry, it is important to understand the behavior of the retained SOV in the subpopulations of patients within these diagnoses: AS versus AI in both BAV and TAV cases. For this reason, we stratified the study population into 4 groups to obtain definitive assessment of potential dilatation of the SOV segment in the context of a defined surgical repair. The overall results of our study strongly support that AVRSCAAR maintains SOV stability over long-term follow-up irrespective of aortic valvular pathology or anatomic valve type.

The majority of investigations on the behavior of the sinus segment have been in patients with BAV. Previous studies have proposed that the sinus segment in BAV aortopathies is at risk for aortic events ${ }^{12,13}$ and suggested

TABLE 7. Cox regression model: Factors associated with mortality over long-term follow-up

\begin{tabular}{lccc}
\hline \multicolumn{1}{c}{ Variable } & HR & $\mathbf{9 5} \%$ CI & $\boldsymbol{P}$ value \\
\hline Age & 1.061 & $1.008-1.117$ & .022 \\
Ascending aorta $(\mathrm{mm})$ & 1.195 & $0.830-1.719$ & .338 \\
Surgical period $\geq 2009$ & 0.275 & $0.113-0.672$ & .005 \\
Gender (male) & 1.201 & $0.524-2.755$ & .665 \\
Hypertension & 1.058 & $0.377-2.969$ & .915 \\
NYHA III/IV & 3.220 & $1.463-7.084$ & .004 \\
SOV >45 mm & 1.040 & $0.286-3.780$ & .952 \\
Valve morphology (TAV/BAV) & 2.407 & $0.946-6.124$ & .065 \\
\hline
\end{tabular}

Day 3: Fate of remnant SOV in patients undergoing aortic valve replacement, ascending aorta replacement, and aortic arch replacement. $H R$, Hazard ratio; $C I$, confidence interval; $N Y H A$, New York Heart Association; SOV, sinus of Valsalva; TAV, tricuspid aortic valve; $B A V$, bicuspid aortic valve. removal of the sinus segment in patients with aortopathies with moderate sinus dilation. ${ }^{12,13}$ Recently, a slower growth rate for the sinus segment and a less-aggressive aortic event rate have been recognized. ${ }^{3,18} \mathrm{~A}$ recent investigation reported no significant change in the sinus segment less than $40 \mathrm{~mm}$ and no difference in survival in patients with BAV between those in whom the sinus segment is removed (Bentall) and those in whom the sinus segment is retained (AVRSCAAR). ${ }^{11}$ In this study, analysis of the sinus segment showed a significant decrease in size in patients with a preoperative sinus segment greater than $40 \mathrm{~mm}$. However, this study did not account for time bias. ${ }^{11}$ A novel concept postulated by Della Corte and colleagues ${ }^{19,20}$ advocates a therapeutic approach to the sinus segment based on BAV leaflet fusion morphology as a method of risk stratification for sinus or ascending aortopathies. A group from the Massachusetts General Hospital recently compared the ascending aorta of patients with BAV and TAV. Their results indicated that TAV ascending aortic segments have more severe histologic changes than BAV, including atherosclerotic changes, elastic fiber and vascular smooth muscle cell loss, as well as medial fibrosis. ${ }^{8}$ These findings advocate for a more moderate approach to BAV aortopathies. ${ }^{8} \mathrm{~A}$ similar investigation comparing the ascending aorta of patients with BAV and TAV found a correlation between root parameters and ascending aortopathies. These ascending aortopathies demonstrated that in the subpopulation of AS, the proximal aorta of TAV had similar behavior as the proximal aorta of $\mathrm{BAV}^{21}$ Overall, the pendulum seems to swing increasingly toward preservation of the moderately dilated sinus segment in BAV and TAV cases.

The results of our study support these investigations, advocating for a more moderate approach to the SOV in bicuspid and tricuspid aortopathies. To validate this strategy, our results suggest an early significant reduction in SOV diameter from preoperative baseline to early postoperative for all patient subgroups $(P<.001)$. Furthermore, unlike other investigations, ${ }^{10,11}$ our study used a repeatedmeasure, mixed-effects model for longitudinal echocardiographic data assessment. This mixed-effects model, which includes aortic valve pathology and morphology, preoperative SOV diameters, postoperative measurement time points, and an interaction term of preoperative SOV with postoperative time as covariates to the longitudinal dataset, suggests that for all follow-up time points (discharge to $\geq 10$ years), the retained SOV dimensions remain stable (within-subject comparison) over long-term follow-up, irrespective of valvular morphology and pathology (Table 6 and Figure 2). We believe that using these results in combination with the decision algorithm detailed in Figure 1 might provide guidance in the decision to retain or resect the sinus segment (AVRSCAAR vs Bentall procedure) in patients with ascending aortopathies with valvular disease. 


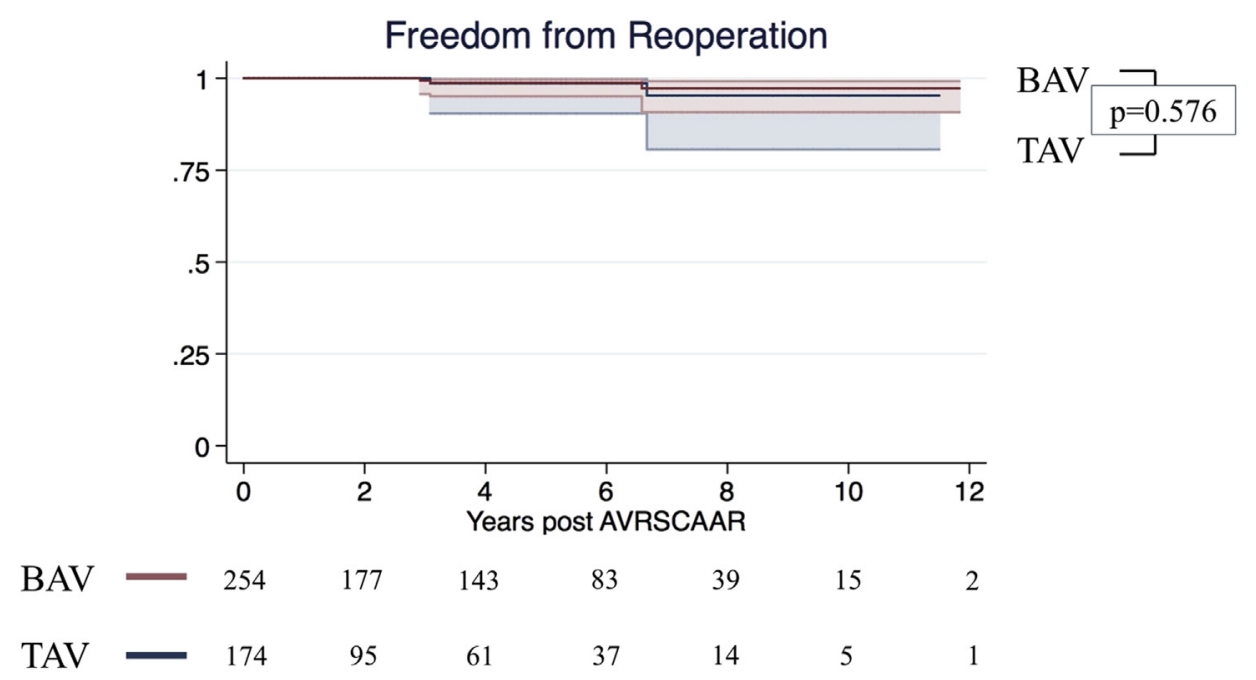

FIGURE 3. Kaplan-Meier freedom from reoperation in BAV and TAV groups. BAV, Bicuspid aortic valve; $T A V$, tricuspid aortic valve; $A V R S C A A R$, aortic valve and supracoronary ascending aortic replacement.

Also, in concordance with the findings of others, the BAV and TAV subpopulations had a high freedom from reoperation. No difference in subgroups was discernible on logrank statistics. There were no surgical aortic events in the sinus segment throughout the follow-up period. Although survival estimates suggested a significant difference in outcomes $(P<.001)$, this most likely indicates a statistical artifact because both groups were not different on attempted propensity score matching after adjusting for age, gender, and renal function $(P=.934$; data not shown). Our cohort size prevented a full propensity score matching. Likewise, we also noted excellent midterm to long-term survival in BAV and TAV cases undergoing AVRSCAAR. Cox regression identified advanced age and advanced heart failure as predictors for mortality. When era of surgery was introduced as a covariate, better survival outcomes were seen in patients undergoing AVRSCAAR after 2009, compared with the period before. Although the surgical technique and the principal surgeons performing the procedure have not changed during the study period, we believe that the mortality difference noted may be a reflection of the increasing experience and volume of AVRSCAAR procedures at our institution. Furthermore, significant advances have been made in the past decade in the postoperative care of patients undergoing cardiac surgery. Our group is looking into this in greater detail to better understand the factors associated with improved outcomes at our center during the more recent time period of the study.

The overall findings in our study may be rationalized by 3 concepts in relation to the SOV: (1) developmental differences between SOV and ascending aorta; (2) pressure/flow changes associated with AVRSCAAR; and (3) changes in aortic root geometry associated with AVRSCAAR. Sinus segment stabilization may be facilitated because of its different embryologic origin compared with the ascending aorta. It has been shown by cell fate mapping studies that the vascular smooth muscle cells of the ascending aorta are of neural crest origin, whereas studies suggest the aortic root develops from contributions of vascular smooth muscle cells from the secondary heart field. ${ }^{15,16,22}$ The STJ is the potential interdigitation transition area of the developmental field boundaries of the vascular smooth muscle cells from the secondary heart field and neural crest origin. ${ }^{16,22,23}$ The different origin of vascular smooth muscle cells in the SOV may enable better sinus segment stabilization even for the moderately dilated aortic root, compared with the ascending aorta. The second concept for sinus segment stabilization invokes the role of transvalvular flow jets and potential rheology-associated hemodynamic shear stress eliciting possible endothelial mechanotransduction, which contributes to aneurysm formation. ${ }^{24-27}$ This shear stress may be reduced with removal of the dysfunctional aortic valve with AVRSCAAR, and in turn may stabilize the sinus segment. ${ }^{24-27}$ The third concept, root geometry, may be a factor in the stability of the retained sinus segment in patients undergoing AVRSCAAR by affecting the mechanical properties of the aortic root. ${ }^{26-28}$ Stabilization of the aortic annulus with a normally functioning aortic valve and reestablishing a STJ/annular ratio with the supracoronary tube graft might restore a more stable geometry of the SOV. This "normalization" of the STJ to annular ratio may stabilize the aortic sinus segment hemodynamics and geometry, and reduce shear stress. ${ }^{26-30}$

\section{Study Limitations}

Although extensive work was performed to obtain echocardiographic data for long-term outcome analysis of the $\mathrm{SOV}$, insufficient data were available for patients with preoperative SOV greater than $45 \mathrm{~mm}$ because of cardiology 

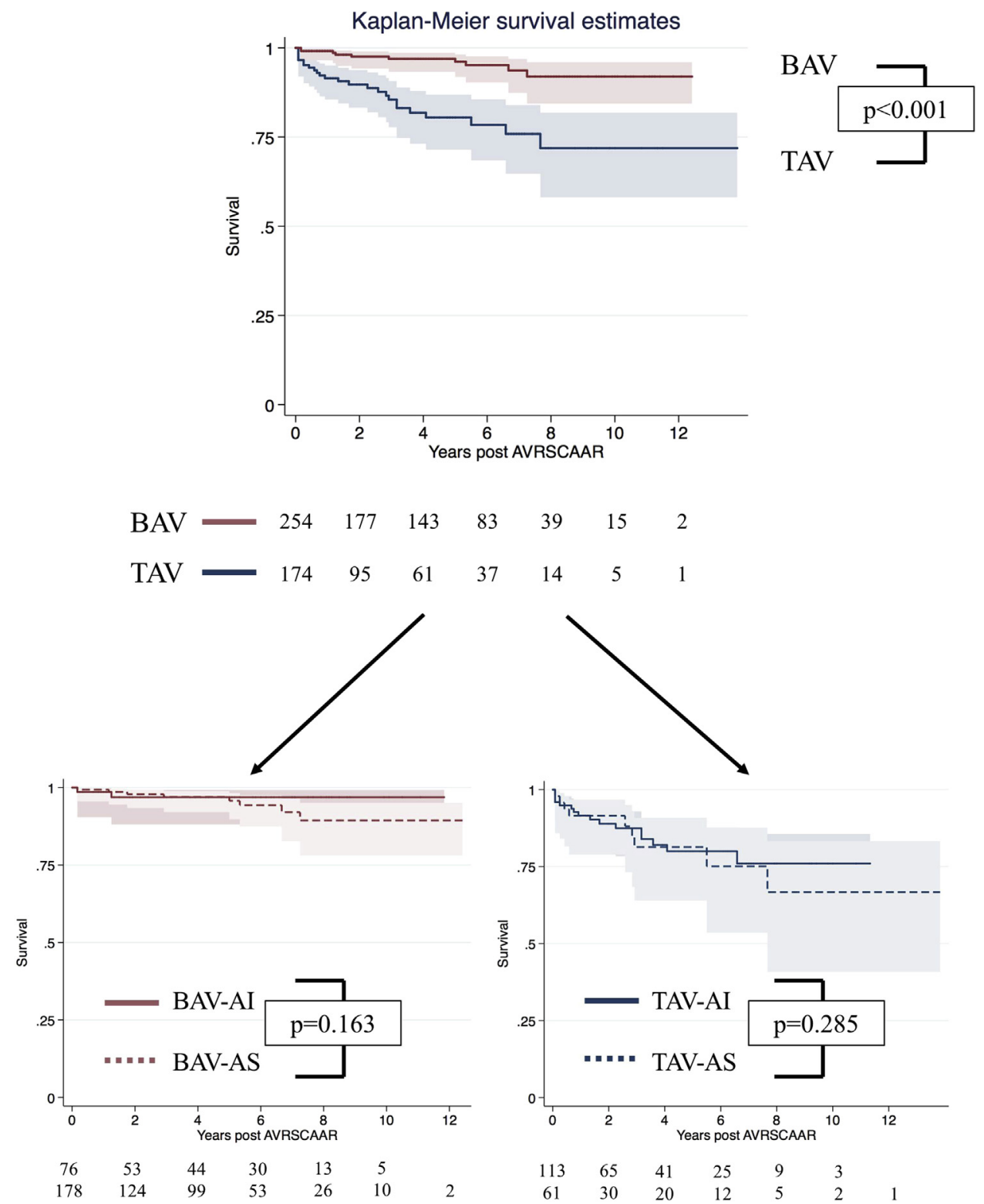

$\begin{array}{llll}83 & 39 & 15 & 2 \\ 37 & 14 & 5 & 1\end{array}$

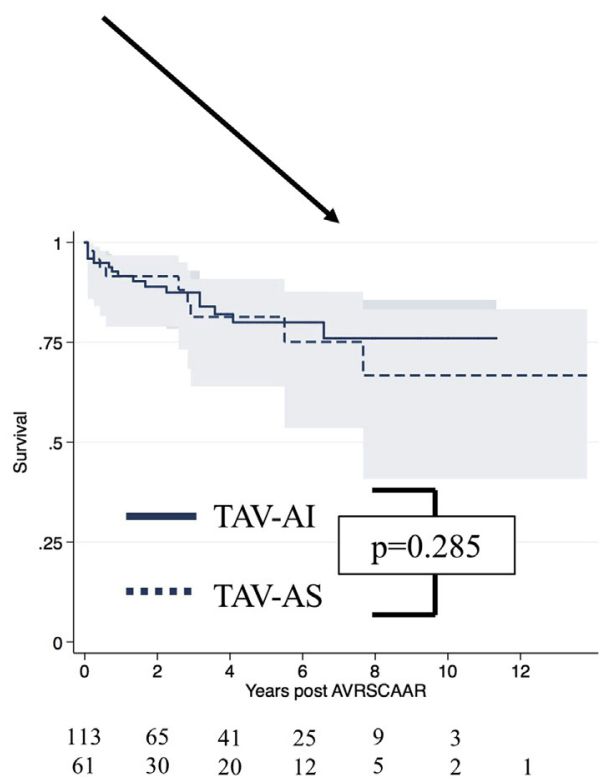

FIGURE 4. Reoperation-free Kaplan-Meier survival in BAV versus TAV groups, and reoperation-free survival within each group by valve pathology (AS vs AI). $B A V$, Bicuspid aortic valve; TAV, tricuspid aortic valve; $A V R S C A A R$, aortic valve and supracoronary ascending aortic replacement; $A I$, aortic insufficiency; $A S$, aortic stenosis.

referral patterns and patient and cardiologist preference for local echocardiographic follow-up. Echocardiograms were not read by one individual, which may introduce operator and reader bias. However, we did make every possible attempt to reach out to all patients and their primary care physicians and cardiologists to obtain complete follow-up data. Missing data were addressed by fitting a mixedeffects, repeated-measure model for longitudinal data assessment and by carefully validating that data were missing at random. Finally, the overall cohort size was not large enough to perform reliable propensity score matching, which we hope to perform in the future with a larger sample size.

\section{CONCLUSIONS}

Our results have shown that for patients undergoing AVRSCAAR, the sinus segment remains stable in all TAV (TAV AS/TAV AI) and BAV (BAV AS/BAV AI) subpopulations. Therefore, irrespective of the aortic valve morphology or valve pathology, in patients with mild to moderate aortic root dilatation $(<45 \mathrm{~mm})$, preservation of the SOV segment in the context of an AVRSCAAR procedure is justified. Continued further follow-up will be important to understand the long-term outcomes of sinus preservation, especially in the younger population with BAVs. 


\section{Conflict of Interest Statement}

Authors have nothing to disclose with regard to commercial support.

\section{References}

1. Fedak PW, Verma S, David TE, Leask RL, Weisel RD, Butany J. Clinical and pathophysiological implications of a bicuspid aortic valve. Circulation. 2002; 106:900-4.

2. Hahn RT, Roman MJ, Mogtader AH, Devereux RB. Association of aortic dilation with regurgitant, stenotic and functionally normal bicuspid aortic valves. $J$ Am Coll Cardiol. 1992;19:283-8.

3. Michelena HI, Della Corte A, Prakash SK, Milewicz DM, Evangelista A, Enriquez-Sarano M. Bicuspid aortic valve aortopathy in adults: incidence, etiology, and clinical significance. Int J Cardiol. 2015;201:400-7.

4. Siu SC, Silversides CK. Bicuspid aortic valve disease. J Am Coll Cardiol. 2010; 55:2789-800.

5. Borger MA, Preston M, Ivanov J, Fedak PW, Davierwala P, Armstrong S, et al. Should the ascending aorta be replaced more frequently in patients with bicuspid aortic valve disease? J Thorac Cardiovasc Surg. 2004;128:677-83.

6. Yasuda H, Nakatani S, Stugaard M, Tsujita-Kuroda Y, Bando K, Kobayashi J, et al. Failure to prevent progressive dilation of ascending aorta by aortic valve replacement in patients with bicuspid aortic valve: comparison with tricuspid aortic valve. Circulation. 2003;108(Suppl 1):II291-4.

7. Tadros TM, Klein MD, Shapira OM. Ascending aortic dilatation associated with bicuspid aortic valve: pathophysiology, molecular biology, and clinical implications. Circulation. 2009;119:880-90.

8. Heng E, Stone JR, Kim JB, Lee H, MacGillivray TE, Sundt TM. Comparative histology of aortic dilatation associated with bileaflet versus trileaflet aortic valves. Ann Thorac Surg. 2015;100:2095-101.

9. Girdauskas E, Borger MA. Bicuspid aortic valve and associated aortopathy: an update. Semin Thorac Cardiovasc Surg. 2013;25:310-6.

10. Park CB, Greason KL, Suri RM, Michelena HI, Schaff HV, Sundt TM III. Fate of nonreplaced sinuses of Valsalva in bicuspid aortic valve disease. J Thorac Cardiovasc Surg. 2011;142:278-84.

11. Vendramin I, Meneguzzi M, Sponga S, Deroma L, Cimarosti R, Lutman C, et al. Bicuspid aortic valve disease and ascending aortic aneurysm: should an aortic root replacement be mandatory dagger. Eur J Cardiothorac Surg. 2016;49:103-9.

12. Etz CD, Homann TM, Silovitz D, Spielvogel D, Bodian CA, Luehr M, et al. Long-term survival after the Bentall procedure in 206 patients with bicuspid aortic valve. Ann Thorac Surg. 2007;84:1186-94.

13. Reece TB, Singh RR, Stiles BM, Peeler BB, Kern JA, Tribble CG, et al Replacement of the proximal aorta adds no further risk to aortic valve procedures. Ann Thorac Surg. 2007;84:473-8.

14. Girdauskas E, Disha K, Borger MA, Kuntze T. Long-term prognosis of ascending aortic aneurysm after aortic valve replacement for bicuspid versus tricuspid aortic valve stenosis. J Thorac Cardiovasc Surg. 2014;147:276-82.

15. Jiao J, Xiong W, Wang L, Yang J, Qiu P, Hirai H, et al. Differentiation defect in neural crest-derived smooth muscle cells in patients with aortopathy associated with bicuspid aortic valves. EBioMedicine. 2016;10:282-90.

16. Waldo KL, Hutson MR, Ward CC, Zdanowicz M, Stadt HA, Kumiski D, et al. Secondary heart field contributes myocardium and smooth muscle to the arterial pole of the developing heart. Dev Biol. 2005;281:78-90.

17. Keane MG, Wiegers SE, Plappert T, Pochettino A, Bavaria JE, Sutton MG. Bicuspid aortic valves are associated with aortic dilatation out of proportion to coexistent valvular lesions. Circulation. 2000;102:III35-9.

18. Gagne-Loranger M, Dumont E, Voisine P, Mohammadi S, Dagenais F. Natural history of 40-50 $\mathrm{mm}$ root/ascending aortic aneurysms in the current era of dedicated thoracic aortic clinics. Eur J Cardiothorac Surg. 2016;50:562-6.

19. Della Corte A, Bancone C, Buonocore M, Dialetto G, Covino FE, Manduca S, et al. Pattern of ascending aortic dimensions predicts the growth rate of the aorta in patients with bicuspid aortic valve. JACC Cardiovasc Imaging. 2013;6: 1301- 10 .

20. Della Corte A, Bancone C, Dialetto G, Covino FE, Manduca S, D'Oria V, et al. Towards an individualized approach to bicuspid aortopathy: different valve types have unique determinants of aortic dilatation. Eur J Cardiothorac Surg. 2014;45: e118-24.

21. Girdauskas E, Rouman M, Disha K, Fey B, Dubslaff G, Theis B, et al. Functional aortic root parameters and expression of aortopathy in bicuspid versus tricuspid aortic valve stenosis. J Am Coll Cardiol. 2016;67:1786-96.
22. Majesky MW. Developmental basis of vascular smooth muscle diversity. Arterioscler Thromb Vasc Biol. 2007;27:1248-58.

23. Lindsay ME, Dietz HC. Lessons on the pathogenesis of aneurysm from heritable conditions. Nature. 2011;473:308-16.

24. Barker AJ, Markl M, Burk J, Lorenz R, Bock J, Bauer S, et al. Bicuspid aortic valve is associated with altered wall shear stress in the ascending aorta. Circ Cardiovasc Imaging. 2012;5:457-66.

25. Humphrey JD, Schwartz MA, Tellides G, Milewicz DM. Role of mechanotransduction in vascular biology: focus on thoracic aortic aneurysms and dissections. Circ Res. 2015;116:1448-61.

26. Azadani AN, Chitsaz S, Matthews PB, Jaussaud N, Leung J, Tsinman T, et al. Comparison of mechanical properties of human ascending aorta and aortic sinuses. Ann Thorac Surg. 2012;93:87-94.

27. Katayama S, Umetani N, Sugiura S, Hisada T. The sinus of Valsalva relieves abnormal stress on aortic valve leaflets by facilitating smooth closure. J Thorac Cardiovasc Surg. 2008;136:1528-35.

28. David TE, Feindel CM, Armstrong S, Maganti M. Replacement of the ascending aorta with reduction of the diameter of the sinotubular junction to treat aortic insufficiency in patients with ascending aortic aneurysm. J Thorac Cardiovasc Surg. 2007; 133:414-8.

29. Kunzelman KS, Grande KJ, David TE, Cochran RP, Verrier ED. Aortic root and valve relationships. Impact on surgical repair. J Thorac Cardiovasc Surg. 1994; 107:162-70.

30. Northrup WF III, Rollins SD. Aortic annuloplasty. In: Yankah CA, Weng Y, Hetzer R, eds. Aortic Root Surgery. Vol 1. 1st ed. Berlin, Germany: Heidelberg Springer-Verlag; 2010:149.

Key Words: aortic root surgery, aortic aneurysm, bicuspid aortic valve, aortic valve surgery

\section{Discussion}

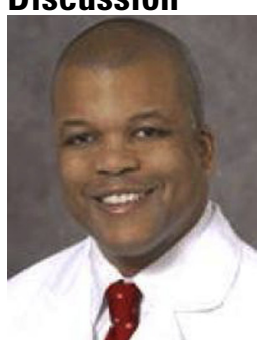

MODERATOR: Dr David Cook

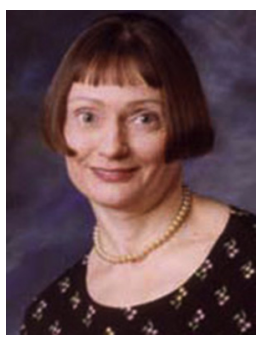

PRESENTER: Dr Rita Milewski

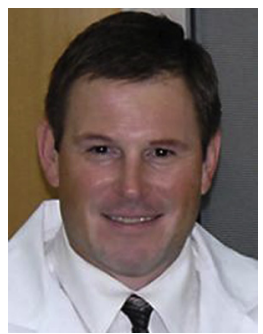

DISCUSSANT: Dr John S. Ikonomidis

Dr David Cook (Davis, Calif). We're going to start Scientific Session Five. We need the program committee to meet at the front of the room at the end of the business meeting to take care of important selections for the meeting. 
We're going to start with Bavaria and colleagues for the Penn group for "Fate of Remnant Sinuses of Valsalva in Patients Undergoing Aortic Valve Replacement, Ascending Aorta, and Aortic Arch Replacement" with Dr Milewski.

Dr Rita Milewski (Philadelphia, Pa). The congenital bicuspid valve and idiopathic trileaflet aortic valve, ascending aortic aneurysms, have a common presentation and manifestation; however, the clinical presentation and pathophysiology are divergent. BAV is the most common congenital valvular problem with syndromic aortopathy. Trileaflet aortic valve idiopathic aortopathy is primarily associated with older age, hypertension, and increased atherosclerotic burden. In patients with critical aortic valve disease and ascending aortic aneurysms, the management of the SOV remains unresolved. Aortic valve replacement and supracoronary ascending aortic replacement have been used for patients with mild to moderate sinus segment dilatation in both TAV and BAV cases. On the basis of studies of the ascending aorta, it has been postulated that patients with BAV have a higher rate of complications of the remaining aorta, but recent clinical and histologic studies have brought this approach into question. BAV and TAV aortopathy are heterogeneous disease processes with a common clinical presentation of a bicuspid or a trileaflet aortic valve. To understand the impact of a common therapeutic surgical approach such as aortic valve replacement and supracoronary ascending aortic replacement on the clinically important aspects of sinus segment, size, and geometry, it's important to understand the behavior of the retained sinus segment in subpopulations of patients with these diseases (eg, AS and AI).

We stratified the study population to 4 subgroups to obtain a definitive assessment of the potential dilatation of the SOV segment in the context of a defined surgical procedure. To date, a direct comparison of the study of the stability of the retained segment between BAV and TAV cases is lacking. We investigated the midterm outcomes and preserved SOV segment stratified by the aortic valve type and pathology. From 2002 to 2015, 428 patients underwent aortic valve replacement and supracoronary ascending aortic replacement electively for valvular and aneurysmal pathology. Median clinical follow-up was 43.5 months (range, 1-166 months). Patients with no connective tissue disease, type A dissection, endocarditis, or reoperations, and emergency cases were excluded. The patients all received aortic valve replacements and $100 \%$ Dacron ascending hemiarch or total arch procedures. All aortoplasties were excluded. To account for the heterogeneous disease processes of those with BAV and trileaflet with aortic valvulopathy, the ascending aneurysm, we stratified the heterogeneous patient population by the following variables: valve morphology, BAV versus TAV, valve pathology, AS versus AI, size of the sinus segment less than 40 to 45 or greater than $44 \mathrm{~mm}$, and the time from the procedure, preoperatively, discharge, less than 2 years, and greater than 2 years. The sinus segment size was measured preoperatively and postoperatively by echocardiography, and the patients were followed in the Aortic Clinic. Despite the greatest efforts, full imaging was not available on all patients for follow-up, and these patients were excluded from the analysis. Preoperative variables revealed that for those with TAV and trileaflet and aortic valves, there was a significant increase or significant difference in age in those with TAV and those with hypertension and dyslipidemia. There was a greater incidence of male gender in those with BAV. Those with trileaflets also had a decreased ejection fraction and an NYHA classification of 3 to 4 at a greater incidence than BAV. There was a greater incidence of AS in those with BAV and AI in those with TAV. Preoperative SOV was stratified between the TAV and BAV subpopulations: less than $40 \mathrm{~mm}, 40$ to $45 \mathrm{~mm}$, and greater than $45 \mathrm{~mm}$. Intraoperative variables revealed significantly longer cardiopulmonary bypass and aortic crossclamp times for TAV cases secondary to increased concomitant coronary artery bypass. Postoperatively, there was an increase in stroke in the TAV cases. These were all embolic strokes and probably secondary to the increased atherosclerotic burden. There was also an increase in renal failure, prolonged ventilation, and in-hospital mortality in the TAV subpopulation. Left ventricular end-diastolic dimensions were decreased in the TAV AI and the BAV AI groups on long-term follow-up. The SOV measurements were obtained for the TAV and BAV subpopulations stratified by the SOV measurements $(<40 \mathrm{~mm}, 40-45 \mathrm{~mm}$, and $>45 \mathrm{~mm}$ ). To eliminate time bias, follow-up SOV dimensions were obtained in 4 time-frames (preoperatively, discharge, $<2$ years, and $>2$ years).

For patients with a preoperative SOV between 40 and $45 \mathrm{~mm}$, there was no difference between preoperative and long-term follow-up SOV size for any of the subgroups, BAV AI or AS, or TAV AI or AS. However, for patients with an SOV less than preoperatively, there was a tendency to a decrease in SOV size between discharge and 2 years; however, after 2 years, the SOV was stabilized to the preoperative size and remained so continuously in follow-up. Freedom from reoperation was high in the BAV group at 10 years. Freedom from reoperation was $97 \%$. In the $\mathrm{TAV}$ group, it was $95 \%$. There were no surgical aortic events in the sinus segment through the follow-up period. Survival for those with TAV was significantly less than for those with BAV, correlating with increased age, atherosclerotic burden, increased coronary artery disease, decreased ejection fraction, and increased incidence of NYHA classification 3 and 4 in the TAV group. Multivariable regression analysis showed that age, NYHA classification 3 and 4, and trileaflet aortic valve morphology were all significant predictors of long-term survival. The findings in our study 
may be rationalized or postulated by 3 hypotheses: developmental differences, pressure flow changes, and aortic root geometry. The sinus segment stabilization may be facilitated because of its different embryologic origin compared with the ascending aorta. It has been shown by cell-fate mapping studies that the vascular smooth muscle cells of the ascending aorta are of neural crest origin where the aortic root develops from contributions of vascular smooth muscle cells from the secondary heart field. The STJ is the interdigitation of these 2 smooth muscle cell origins, and the difference in vascular smooth muscle cell in the sinus segment may contribute to this aortic stabilization. Aortic valve replacement and supracoronary ascending aortic replacement may decrease or remove the role of hemodynamic shear and mechanical transduction, and may allow vascular smooth muscle cells to return to a contractile from a synthetic phenotype and allow remodeling of the sinus segment. Stabilizing the aortic annulus with a normally functioning prosthetic aortic valve and reestablishing the STJ to annular ratio with a supracoronary tube graft may restore the stable geometry to the SOV, allowing the vascular smooth muscle cells and the sinus segment itself to remodel to a normal geometry.

Our results have shown that for patients who received aortic valve replacement and supracoronary ascending aortic replacement, the sinus segment remains stable in all TAV, TAV AS and AI, and BAV AS and AI subpopulations. Therefore, irrespective of the aortic valve morphology or valve pathology in patients with mild to moderate aortic root dilatation, preservation of the SOV segment in the context of an aortic valve replacement and supracoronary ascending aortic valve replacement procedure is justified.

Dr John S. Ikonomidis (Chapel Hill, NC). There are sufficient data in the literature to indicate that aortopathy related to BAVs may be influenced by cusp fusion pattern. The most common, I guess, $70 \%$ to $75 \%$, is left-to-right, and there are some data indicating this is also associated with the most aggressive remodeling in the ascending aorta, and perhaps also in the aortic root, and I was wondering if you had considered stratifying your results by cusp fusion pattern?

Dr Milewski. Yes, I' $m$ familiar with these studies such as the recent article by Della Corte and colleagues. Stratification on the basis of BAV leaflet fusion morphology would add interesting data to the study and we actually considered this stratification. However, because this is a retrospective study, delineation of BAV leaflet fusion morphology was not available on many of our preoperative echocardiograms.

Dr Ikonomidis. Moving forward, are you documenting this?

Dr Milewski. Yes, we plan going forward to incorporate this into our future BAV studies.
Dr Ikonomidis. What is your method of imaging followup? Is it echocardiography, magnetic resonance imaging/ computed tomography, or a mix of these?

Dr Milewski. Some are mixed if the patients have any residual aortic pathology. We perform echocardiograms on all of our patients. We obtain an echo preoperatively, obviously, and intraoperative transesophageal echocardiograms are performed as well as an echo prior to discharge. Followup studies are by echocardiography for aortic valve and cardiac function as well as proximal aortic sinus segment dimensions. If patients have any kind of residual aortic pathology, they are followed up by computed tomography angiography as well. We don't routinely follow-up by magnetic resonance imaging or angiography.

Dr Ikonomidis. Are you concerned about the ability of transthoracic echocardiograms to accurately discriminate root dimensions?

Dr Milewski. Patients are referred into our aortic clinic from a wide geographic area and many patients prefer to be followed up by local cardiologists and have locally performed follow-up studies. Unlike centers in which all patients are seen by in-house cardiologists postoperatively, many of our patients are limited geographically and follow up with a local cardiologist. Therefore, in our aortic clinic, we rely on outside cardiologists to perform and read follow-up echocardiograms, so, to address your question, yes there are occasionally some interreader differences in echocardiogram root dimensions, which may be a minor limitation.

Dr T. Brett Reece (Aurora, Colo). Michael Borger wrote an article not too long ago that said if you put in more than a 25-0-mm valve, the annulus dilated, and they thought the roots were going to fall apart in those patients and had some follow-up to suggest this. People would receive reroot replacement. Do you have any proxies of size or anything that would be in addition to this that might give us an idea that there's more degeneration of the root than just the size of the sinuses or STJ?

Dr Milewski. No. We routinely use the largest valve that is appropriate. Most of our patients were normalized to a STJ/annular ration of $1: 1$ by using supracoronary Dacron graft, establishing a more normal geometry to the aortic root. We did not identify any root deterioration.

Dr Joseph E. Bavaria (Philadelphia, Pa). I don't believe that article by the way. I would say I didn't see our data there, but I'm willing to bet a significant amount of money that our average size of a valve is at least 25 to $27 \mathrm{~mm}$.

Dr Milewski. Actually, Joe, you're right.

Dr Bavaria. In this group, we used big valves and didn't really worry about that valvular annular concept, and I think the surrogate is AI. The patients who have AI who showed no difference almost always had big valves. 